\title{
The Branch of Theoretical Analysis and Application Research on Grid String Structure
}

\author{
ZHANG Jiarui* \\ Class 1311, Chengguan middle school Wuqing,Tianjin, 301700,China
}

\begin{abstract}
According to the ideas of the beam string structure, to stress structure is put forward, this is a very flexible cable for joined the rigid grid and support.This is the grid string branch structure.String branch established grid computing model.Static and dynamic characteristics of structure, it shows the different prestressing is analyzed.Through the analysis of structure displacement and internal force of the rod is according to the prestressing value obviously improved.Prestress value is larger, the structure displacement and internal force of the lever is low.The characteristic of structural natural vibration almost no change, although prestress value is changing. When the structure analysis, the structural vibration response curve conforms to the general law. Node displacement response and lower chord node block effect did not happen.
\end{abstract}

\section{Introduction}

Topics in prestressed steel structure research about 60 years from now. Compared with non - prestressed steel structure, saves materials and steel consumption can be reduced by using prestressed [1].Consumption and use different types of prestressed steel structure material is different. Now different types of prestressed steel structure including traditional type, suspension type, stretching integral type, beam string structure type, cable and membrane type, glass curtain wall type string.

Beam string structure type is a kind of space systems, it is according to the development of plane beam string. The reinforcement structure is made up of intermediate support, formed by reinforcement of prestressed cable and pillar. Originally a form of prestressed steel structure reinforcement., lower chord connecting by struts on members of the rigid and flexible. Load advantages of reasonable structure, light weight, processing and transportation is convenient.

According to the ideas of the beam string structure, to support its compressive stress structure put forward the continuous tensile and compression components. It is a string $(2,3$, 4) branch structure. It is grid string branch structure, grid and rigidity of soft cable connection rod support $(5,6,7)$.In this article, a grid string branch structure model was established. Static and dynamic characteristics of structure, it shows the different prestressing is analyzed. Nonlinear finite element method (fem) analysis method for mixed

\footnotetext{
*Corresponding author:304507991@qq.com
} 
structure of bar and cable coupling device. Is a comprehensive and general finite element analysis tool ANSYS software.

\section{Static Characteristics Analysis}

The engineering situation. String branch has a grid structure. The plane size $2.5 \mathrm{~m} * 2.5$ $\mathrm{m}$. The four pyramid grid form is positive. Order a one-way cable. Rigid structure with the upper and bottom struts cable connection in the middle. Struts in the midspan is the height of 1.35 meters, 0.8 meters. The end of the cable through the upper chord node and the anchor wall or column. Figure 1 shows the model grid string branch.

The section size of upper chord, members of the network and the support structure is a branch of the $108 \times 4$ string. The section size of bottom chord is $114 \times 5$. The section size of the cable is 515.2.Fu $=1860 \mathrm{mpa}$. All the nodes in the first quarter on concentration. The value is $\mathrm{P}=20 \mathrm{kn}$. Cable prestress value $\mathrm{P} 1=20 \mathrm{kn}, \mathrm{P} 2=40 \mathrm{kn}, \mathrm{P} 3=60 \mathrm{kn}, \mathrm{P} 4=80 \mathrm{kn}$, $\mathrm{P} 5=100 \mathrm{kn}$.

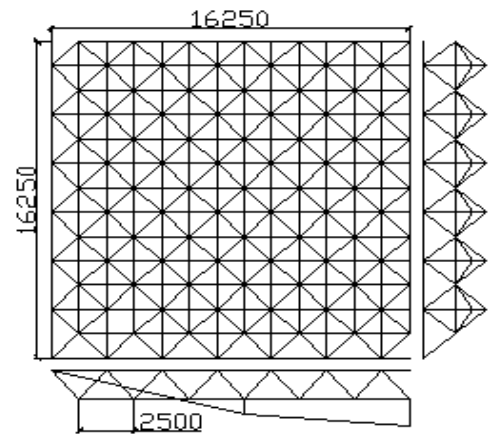

Fig.1 1/4 Model diagram of grid string branch

Static characteristic analysis. Load combination and the calculation results. String on the static characteristics of the branch structure, it is made of different prestress value behavior were studied. As a result, the load combination is:

The first condition is $\mathrm{P}+\mathrm{P} 1$. The second condition is $\mathrm{P}+\mathrm{P} 2$. The third condition is $\mathrm{P}+$ $\mathrm{P} 3$. The fourth condition is $\mathrm{P}+\mathrm{P} 4$. The fifth condition is $\mathrm{P}+\mathrm{P} 5$.

The characteristics of the static model, it is made of five kinds of behavior conditions with finite element software Ansys. Structure of the maximum displacement and stress values, it is made of a variety of conditions such as shown in table 1.

Table 1 The Largest Static Displacement And Stress

\begin{tabular}{|c|c|c|c|c|c|c|}
\hline & \multicolumn{5}{|c|}{ Conditons } \\
\hline & & 1 & 2 & 3 & 4 & 5 \\
\hline \multicolumn{2}{|c|}{$\begin{array}{c}\text { Maximum } \\
\text { Displacement/mm }\end{array}$} & -73.50 & -69.60 & -65.70 & -61.80 & -57.90 \\
\hline \multirow{5}{*}{$\begin{array}{l}\text { Maximum } \\
\text { Stress/MPa }\end{array}$} & Top chord & 116.47 & 109.93 & 103.39 & 96.85 & 90.31 \\
\hline & $\begin{array}{c}\text { Web } \\
\text { member }\end{array}$ & -58.49 & -56.26 & -54.03 & -51.79 & -49.56 \\
\hline & $\begin{array}{l}\text { Bottom } \\
\text { chord }\end{array}$ & 150.22 & 142.34 & 134.47 & 126.60 & 118.73 \\
\hline & Strut & -22.81 & -24.36 & -25.91 & -27.46 & -29.01 \\
\hline & Cable & 198.54 & 212.05 & 225.57 & 239.09 & 252.60 \\
\hline
\end{tabular}


According to the displacement analysis of Table 1, the influence of the structural displacement is greatly influenced by the prestress value. The prestress value is larger, the structure displacement and the internal force of the rod are lower. Therefore, according to the prestress value, the displacement of the structure is obviously improved.

Stress analysis. Top chord. In the string structure of the top string, by the five conditions, some members are under pressure, some of the tension. In the middle part of the structure of the member is compressed, the rest of the other parts of the stretch. The prestress value is bigger, the stress value of the top rod is small. Therefore, the stress of the top string is obviously improved, which depends on the prestress value.

Network member. In the network member of the string branch structure, it is composed of five conditions, some members are compressed and other people are nervous. The stress value of the middle part of the structure is less than the other. The stress value of the network member is small, the stress value is small. Therefore, the pressure of network members is obviously improved, which depends on the prestressing value.

Bottom chord. The bottom chord of the string structure is subjected to tension. Only part of the members are in support of the compression. The maximum stress of the bottom chord is the tensile stress. The stress value of the middle part of the structure is the largest. The prestress value is bigger, the stress value of the bottom chord is small. Therefore, the bottom chord stress is obviously improved, according to the prestress value.

Pillar. All the pillars of the string branch structure, which is compressed by five conditions. The stress values of the middle part of the structure are the largest. Prestress value is bigger, the stress value is bigger. But the magnitude of the increase is not obvious, especially.

Cable All cables are subjected to tensile forces acting on the five condition of the grid string structure. The ultimate stress value of the cable in the middle part of the structure is the largest. The ultimate stress value of the cable is reduced from the middle part to the two sides. The prestressing force is bigger and the ultimate stress value of the cable is larger.

\section{Dynamic Analysis}

Model analysis. First six natural frequency string branch of grid structure, it consists of five conditions is to solve the finite element software Ansys and P3 prestress value of vibration mode. This is shown in figure 2 and table 2.

Table 2 Before Six Order Natural Frequency Of Different Prestressing Value

\begin{tabular}{|c|c|c|c|c|c|c|c|}
\hline & & 1 & 2 & 3 & 4 & 5 & 6 \\
\hline $\mathrm{P}_{1}$ & \multirow{5}{*}{$\mathrm{f} / \mathrm{HZ}$} & 6.591 & 11.667 & 12.085 & 16.621 & 24.159 & 24.496 \\
\hline $\mathrm{P}_{2}$ & & 6.591 & 11.667 & 12.082 & 16.620 & 24.155 & 24.495 \\
\hline $\mathrm{P}_{3}$ & & 6.590 & 11.667 & 12.079 & 16.619 & 24.152 & 24.495 \\
\hline $\mathrm{P}_{4}$ & & 6.589 & 11.668 & 12.076 & 16.618 & 24.149 & 24.494 \\
\hline $\mathrm{P}_{5}$ & & 6.588 & 11.668 & 12.073 & 16.618 & 24.145 & 24.494 \\
\hline
\end{tabular}


According to table 2, string branch of the structure of the natural frequency is intensive. Some adjacent second order frequency values are similar. Natural frequency value decreased significantly, and prestressed value increases. But the natural frequency value is almost no influence by prestress value. According to the figure 2, the first six order vibration mode is vertical. So the grid of the main branch structure of vertical seismic action string.

Time history analysis. Branching structure grid string with tianjin wave time history analysis. Calculation, the maximum vertical acceleration is $75.56 \mathrm{~cm} / \mathrm{s} 2$.Earthquake magnitude is grade seven. The coefficient is 0.81 . The vertical earthquake action is the unique factor. Cable prestress value of P3.Top and bottom of the vertical displacement response of the midspan nodes as shown in figure 3. According to figure 3, conform to the general law of vibration displacement response curve. Top and bottom of the node displacement response curve are the same. As a result, the node displacement response does not occur retardation effect

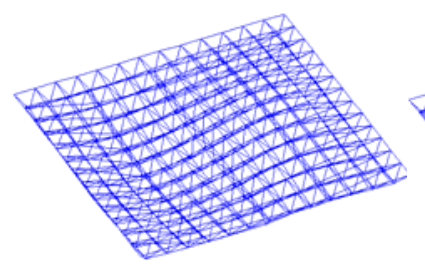

First mode

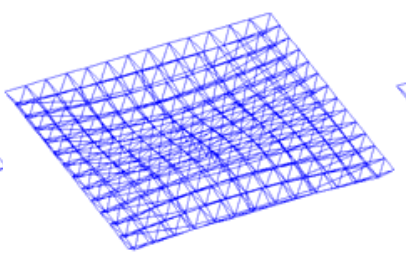

Second mode

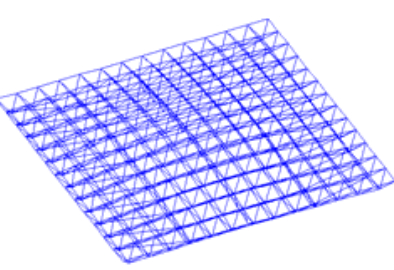

Third mode
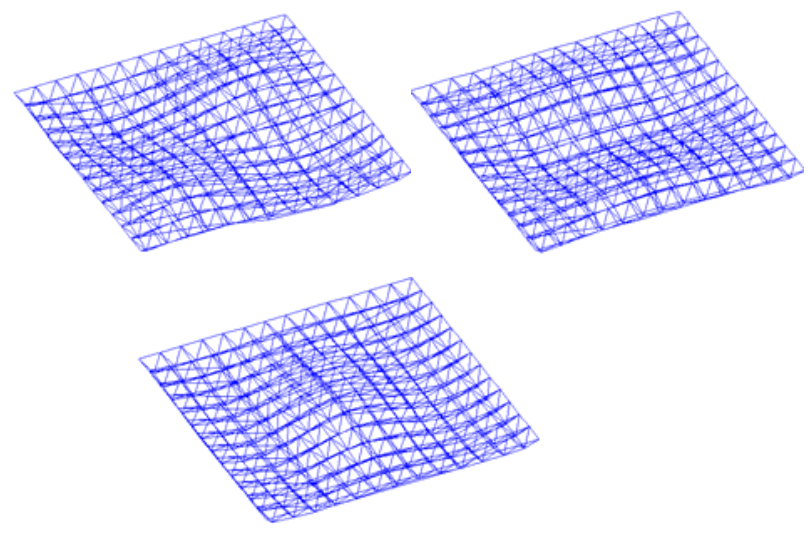

Fourth mode

Fifth mode

Sixth mode

Fig.2 The first six vibration modes 


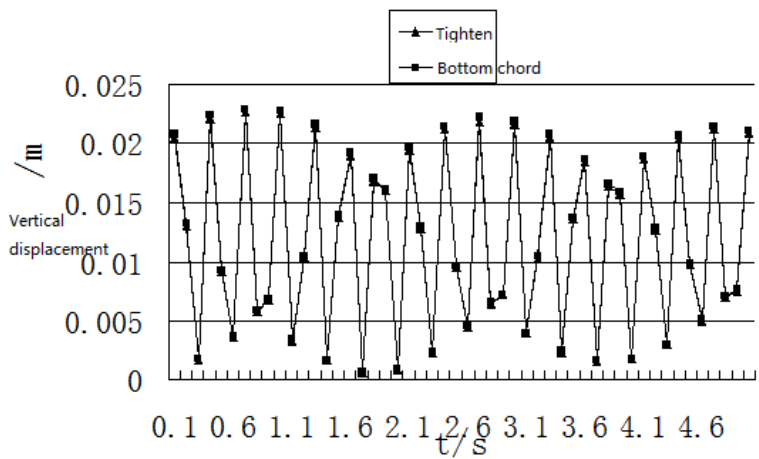

Fig.3 Displacement response of nodes in mid-spon

\section{Summary}

According to analysis of a series of grid string branch structure, there are three conclusions.

(1) the displacement and pressure grid string branch of chord structure obviously improved according to the prestress value. Prestress value is bigger, smaller values of the chord of the displacement and stress.

(2) the natural frequency value is almost no influence by the prestress value. Is just reduced obviously, the prestressing value increases.

(3) Conforms to the general law of vibration displacement response curve. It is not happen the retardation effect.

\section{References}

1. Cilin Lu Yin and west cool horses, liu: modern prestressed steel structure (China newsletter, Beijing 2003).

2. Tsinghua humber pei: submit the building structure (in China, 2004).

3. Tsinghua humber pei: submitted to earthquake engineering and engineering vibration (China, 2004).

4. Chen: wah industrial building (China, 2010).

5. Chen: wah industrial building (China, 2010).

6. Mr Zhang liu: submit industrial buildings (China, 2010).

7. Xiangyu yan: submit industrial buildings (China, 2010).

8. Petroleum ether can P.B. Cachim, V.M. ferreira, the influence of the rheology of fine aggregatThe performance of high performance cement - several silica system.Build.Die.24(2010) 640-2010.

9. B. Friede Microsilica - unique characterization of the additive, journal10th international conference on Inorganic - Bonded fiber composites IIBCC (2006)Sao Paulo, Sao Paulo, razil, and the university of Idaho in October15-2006, pp. 135-144.

10. B fuller, S.E. Thompson proportioning of concrete aw,trans.Points.Soc.Civilization.Eng.33 (1907) 222-1907.

11. Panic, j. d. homers and forecast process control crowded particles in suspensionApplied to ceramics manufacturing, Kluwer academic publishers, Boston, USA,In 1994, pp. 1-765. 